\title{
Sequential Apparent Diffusion Coefficient for Assessment of Tumor Progression in Patients with Low-Grade Glioma
}

(DI.E. Chen, (D) N. Swinburne, (D) N.M. Tsankova, (D) M.M. Hefti, (D)A. Aggarwal, (D)A.H. Doshi, (D) A. Hormigo, (D) B.N. Delman, and $\mathbb{D}_{\mathrm{K} . \mathrm{Nael}}$

\begin{abstract}
BACKGROUND AND PURPOSE: Early and accurate identification of tumor progression in patients with low-grade gliomas is challenging. We aimed to assess the role of quantitative ADC analysis in the sequential follow-up of patients with low-grade gliomas as a potential imaging marker of tumor stability or progression.

MATERIALS AND METHODS: In this retrospective study, patients with a diagnosis of low-grade glioma with at least 12 months of imaging follow-up were retrospectively reviewed. Two neuroradiologists independently reviewed sequential MR imaging in each patient to determine tumor progression using the Response Assessment in Neuro-Oncology criteria. Normalized mean ADC (ADC mean) and 10th percentile $A D C\left(A D C_{10}\right)$ values from FLAIR hyperintense tumor volume were calculated for each MR image and compared between patients with stable disease versus tumor progression using univariate analysis. The interval change of ADC values between sequential scans was used to differentiate stable disease from progression using the Fisher exact test.

RESULTS: Twenty-eight of 69 patients who were evaluated met our inclusion criteria. Fifteen patients were classified as stable versus 13 patients as having progression based on consensus reads of MRIs and the Response Assessment in Neuro-Oncology criteria. The interval change of $A D C$ values showed greater concordance with ultimate lesion disposition than quantitative ADC values at a single time point. The interval change in $A D C_{10}$ matched the expected pattern in 12/13 patients with tumor progression (overall diagnostic accuracy of $86 \%$, $P<.001)$. On average, the $A D C_{10}$ interval change predicted progression 8 months before conventional MR imaging.
\end{abstract}

CONCLUSIONS: The interval change of $A D C_{10}$ values can be used to identify progression versus stability of low-grade gliomas with a diagnostic accuracy of $86 \%$ and before apparent radiologic progression on conventional MR imaging.

ABBREVIATIONS: $L G G=$ low-grade glioma; $R O C=$ receiver operating characteristic

$\mathbf{D}$ iffuse gliomas are the most common type of low-grade gliomas (LGGs), defined by the World Health Organization as grade II diffuse astrocytomas or oligodendrogliomas, most of which have now been found to carry a gain-of-function mutation in the isocitrate dehydrogenase gene. Isocitrate dehydrogenase-mutant diffuse astrocytomas and oligodendrogliomas are relatively slow-growing tumors but inevitably undergo anaplastic transformation into high-grade tumors. ${ }^{1}$ This transformation can manifest differently in the amount of time to progression for each

Received August 30, 2017; accepted after revision February 24, 2018.

From the Departments of Radiology (I.E.C., N.S., A.A., A.H.D., B.N.D., K.N.), Pathology (N.M.T., M.M.H.), and Neurology (A.H.), Icahn School of Medicine at Mount Sinai, New York, New York.

Please address correspondence to Kambiz Nael, MD, Department of Radiology, Icahn School of Medicine at Mount Sinai, Box 1234, One Gustave L. Levy Place, New York, NY 10029; e-mail: Kambiznael@gmail.com; @kambiznael

- Indicates open access to non-subscribers at www.ajnr.org

http://dx.doi.org/10.3174/ajnr.A5639 patient. ${ }^{2}$ Distinguishing LGGs from high-grade gliomas is important for prognosis and guiding treatment. ${ }^{3}$

The Response Assessment in Neuro-Oncology criteria have been established for assessment of progression in patients with LGGs using both clinical (ie, neurologic) and radiologic terms. ${ }^{4}$ In clinically stable patients, progression is likely to be determined radiologically. Imaging surveillance of patients with LGGs is routinely conducted by sequential MR imaging. LGGs are most commonly (>90\%) nonenhancing tumors that are therefore best depicted on FLAIR imaging. Radiologic progression of LGGs is often inferred from an increase in tumor size on FLAIR images or development of enhancement on T1-weighted postcontrast MR imaging. ${ }^{5-8}$ In the absence of new or progressive enhancement, determination of radiologic progression in LGGs might be challenging because slow progression is manifested by small, incremental, and asymptomatic increases in size on FLAIR hyperintense lesion. ${ }^{7}$ Therefore, despite using standardized criteria such as the Response Assessment in Neuro- 


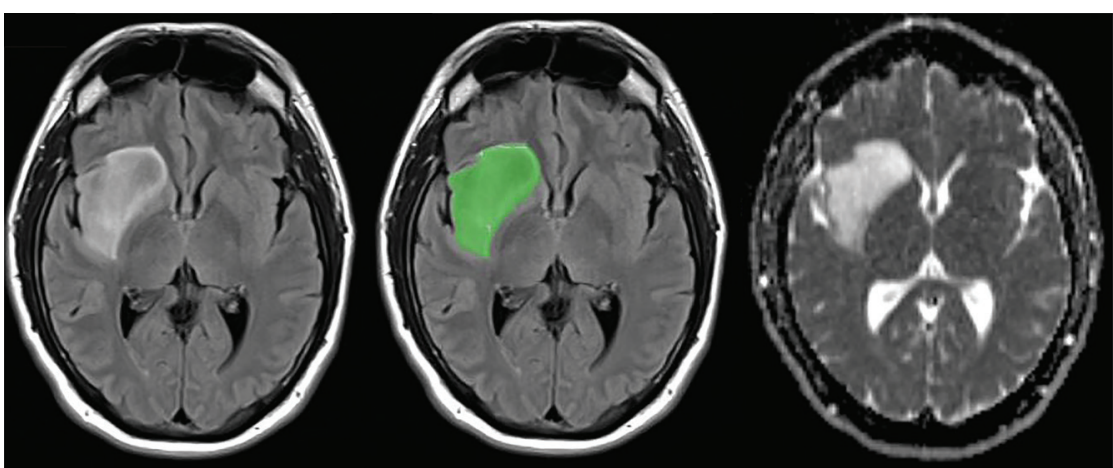

FIG 1. A VOI was generated using a voxel-based signal intensity threshold method subsuming the entire region of FLAIR hyperintensity. Using coregistered images, we transferred the VOI to ADC for quantitative ADC analysis.
T2WI (TR/TE/flip angle, 3100-7000/86$105 \mathrm{~ms} / 180^{\circ}$ ), FLAIR (TR/TE/TI, 800012,000/98-130/2400-2700 ms), DWI, and postcontrast T1-weighted imaging.

DWI was acquired using a singleshot spin-echo EPI sequence (TR/TE, $4900 / 98 \mathrm{~ms}[1.5 \mathrm{~T}]$ or $4100 / 95 \mathrm{~ms}$ [3T]; FOV, $220 \times 220 \mathrm{~mm}$; matrix, $128 \times 128$ $\mathrm{mm}$; slices, $30 \times 5 \mathrm{~mm}$ ). Diffusion gradients were applied along 3 orthogonal directions with $b=0$ and $1000 \mathrm{~s} / \mathrm{mm}^{2}$. A total volume of $0.1 \mathrm{mmol} / \mathrm{kg}$ of gadobenate dimeglumine was injected intravenously for postcontrast T1-weighted imaging.
Oncology, final tumor assignments as stable versus progression may vary greatly among neuroradiologists, associated with intraobserver variability and potential missed diagnoses. ${ }^{9}$

ADC derived from DWI has been shown to reflect tumor cellularity ${ }^{10,11}$ and can have clinical implications for characterizing gliomas. While ADC has been extensively studied to evaluate tumor grade ${ }^{12-15}$ or response to treatment, ${ }^{16,17}$ the literature on the role of $\mathrm{ADC}$ in the assessment of tumor progression in patients with LGGs remains sparse, with mixed results. ${ }^{18}$

More research is necessary to further evaluate the contribution of $\mathrm{ADC}$ in predicting tumor progression in LGGs so that any change in treatment and management may be determined earlier than is possible with conventional sequences. The purpose of this study was to assess the role of quantitative ADC analysis in the sequential follow-up of patients with LGGs as a potential imaging marker of tumor stability or progression.

\section{MATERIALS AND METHODS \\ Patients}

This retrospective study was performed under institutional review board approval with a waiver of informed consent. Digital charts of patients with histologically proved LGGs between 2010 and 2015 were retrospectively reviewed. Inclusion criteria were as follows: 1) a histopathologic diagnosis of grade II diffuse astrocytoma, grade II oligodendroglioma, or grade II glioma with mixed oligodendroglial and astrocytic morphology; 2) no prior interventions except for biopsy or anticonvulsant medications; 3 ) at least 12 months of clinical and imaging follow-up from the date of the operation or biopsy; and 4) availability of diagnostic MR imaging with inclusion of DWI. Study end points were clinical and imaging evidence of progression using the Response Assessment in Neuro-Oncology criteria ${ }^{4}$ or a second surgical resection.

Demographic data including age, sex, initial tumor size, tumor type, extent of the initial surgical resection, duration of follow-up, and number of MRIs were noted for each patient.

\section{Image Acquisition}

MR imaging was performed using 8 MR imaging scanners: 2 Skyra 3T (Siemens, Erlangen, Germany), 2 Aera 1.5T (Siemens), and 4 Signa 1.5T (GE Healthcare, Milwaukee, Wisconsin) within our radiology department. The imaging protocol included transverse T1weighted imaging (TR/TE/flip angle, 600-1800/9-19 ms/180),

\section{Qualitative Image Analysis}

Image analysis was performed in separate reading sessions, independently, by 2 board-certified neuroradiologists who were blinded to the patient outcome. MR imaging studies of each patient were interpreted sequentially by each reader and designated as stable or progressed in comparison with prior MR imaging using the Response Assessment in Neuro-Oncology criteria. ${ }^{4}$ Progression was defined as development of new lesions, increase in enhancement, or increase in the size of a FLAIR hyperintense region by $25 \%$. Discrepancies were resolved by a consensus read. Clinically, progression was defined by definite clinical deterioration related to the tumor or failure to return for follow-up because of death or a deteriorating condition as assessed by the treating neuro-oncologist. Each patient was assigned a final assessment score of "stable" $($ score $=0)$ for patients who remained stable throughout the entire follow-up time and "progressed" $($ score $=1)$ for patients who showed progression.

\section{Quantitative ADC Analysis}

Quantitative ADC analysis was performed using a Food and Drug Administration-approved commercial software package (Olea Sphere; Olea Medical, La Ciotat, France). ADC and FLAIR were coregistered on each examination using a 6- $d f$ transformation and a mutual information cost function. Subsequently, a VOI was generated using a voxel-based signal intensity threshold method subsuming the entire region of FLAIR hyperintensity (Fig 1). From each VOI, a histogram was generated on the basis of voxel-based ADC values. These values were normalized to an ROI placed in the normal-appearing white matter in the contralateral centrum semiovale. Normalized mean $\mathrm{ADC}\left(\mathrm{ADC}_{\text {mean }}\right)$ and 10th percentile $\mathrm{ADC}\left(\mathrm{ADC}_{10}\right)$ values and normalized ratios were exported for statistical analysis. In addition, $\mathrm{ADC}_{\text {mean }}$ and $\mathrm{ADC}_{10}$ ratios were compared between sequential scans in each patient and scored to assess the fit with the expected pattern. An interval decrease of ADC values of $\geq 20 \%$ was an unfavorable pattern, suggestive of progression. Any other pattern, including an increase or a decrease by $<20 \%$, was considered a favorable pattern and suggestive of relative stability. 


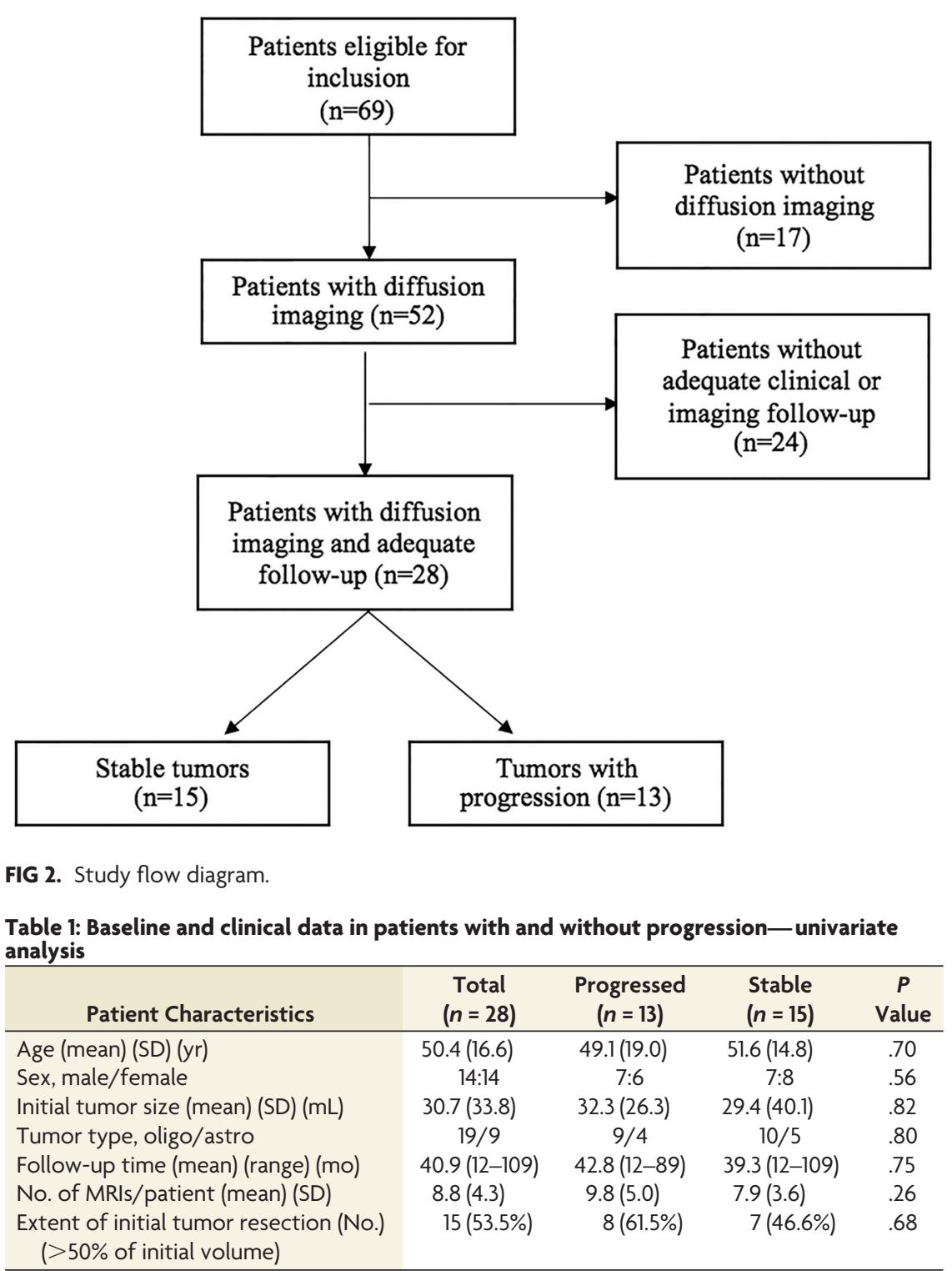

Note:- oligo indicates oligodendroglioma; astro, astrocytoma.

\section{Surgical Resection}

All patients had initial surgical resection to confirm the diagnosis of LGG. The extent of the initial surgical resection was assessed in comparison with the preoperative MR image using the FLAIR volume and dichotomized as follows: 1) core biopsy or limited $(<50 \%$ resection of initial tumor volume), or 2$)>50 \%$ resection of the initial tumor volume. In addition, 13 of 28 patients had a subsequent resection, which was considered an end point in our study. The results of that second surgical pathology were compared with our neuroimaging variables.

\section{Statistical Analysis}

Statistical analysis was performed using MedCalc for Windows (Version 12.2.1; MedCalc Software, Mariakerke, Belgium). Clinical characteristics and neuroimaging variables were compared between subjects with progression and stable disease using univariate analysis with the Fisher exact test for categoric variables and the unpaired 2-tailed Student $t$ test for continuous variables. Interobserver agreement was tested using a linear weighted $\kappa$ test.
Receiver operating characteristic (ROC) analysis was performed to determine the optimal parameters in distinguishing progression from stable disease. Optimal thresholds were calculated for each ROC curve to maximize both sensitivity and specificity using the Youden J statistic.

All lesions were scored into their respective groups without any indeterminate classifications. The patterns of interval changes of $\mathrm{ADC}_{\text {mean }}$ and $\mathrm{ADC}_{10}$ were assessed for diagnostic correlation using the Fisher exact test among patients with progression and stable disease. An interval change of ADC ratios of $>20 \%$ between 2 consecutive studies was considered significant. For each subject, a regression line was generated across multiple MRIs, and a final score of stable versus progression was assigned on the basis of the slope of this line. This was calculated using the average value of $1 \mathrm{SD}$ of the ADC values, which was approximately 0.2 . Accuracy statistics including sensitivity, specificity, and overall diagnostic accuracy were reported. $P<.05$ was used to indicate statistical significance.

\section{RESULTS}

Of 69 patients who were evaluated, 41 patients were excluded due to lack of DWI $(n=17)$ or inadequate clinical or imaging follow-up $(n=24)$ (Fig 2). As a result, 28 patients (14 men and 14 women; mean age, $50.4 \pm 16.6$ years) were included in our analysis. Nineteen patients had oligodendrogliomas (grade II, currently diagnosed as isocitrate dehydrogenase-mutant and 1p/19q-codeleted), and 9 patients had diffuse astrocytomas (grade II, currently diagnosed as isocitrate dehydrogenase-mutant). Fourteen tumors were in the frontal lobe, 6 were in the parietal lobe, 5 were in the temporal lobe, and 3 were in both the frontal and temporal lobes.

A total of $247 \mathrm{MR}$ imaging scans at different time points were evaluated (mean, $8.8 \pm 4.3$ scans per patient). The mean follow-up time was 40.9 months (range, 12-109) months. The final designation obtained from the consensus read of the 2 neuroradiologists showed tumor progression in 13 patients and stable disease in 15 patients.

The patients' age, sex, initial tumor size, tumor type, extent of initial tumor resection, duration of follow-up, and number of MRIs are summarized in Table 1.

\section{Lesion Analysis}

For subjective assessment of disease progression when evaluating conventional imaging (FLAIR and T1-postcontrast), observers 1 
and 2 correctly identified 8/13 (sensitivity, 61.5\%) and 7/13 (sensitivity, 53.8\%), respectively, as tumor progression compared with final assignment. Stable disease was identified in 216/228
(94.7\%) and 222/228 (97.4\%) scans by observers 1 and 2, respectively. The interobserver agreement was $\kappa=0.61$ (95\% CI, $0.4-0.77)$.
Table 2: Diagnostic accuracy of ADC interval change-Fisher exact test

\begin{tabular}{llcccc}
\hline \multirow{1}{*}{ ADC } & Interval Change & $\begin{array}{c}\text { Stable } \\
\text { Tumors } \\
(\boldsymbol{n}=15)\end{array}$ & $\begin{array}{c}\text { Progressed } \\
(\boldsymbol{n}=13)\end{array}$ & $\begin{array}{c}\boldsymbol{P} \\
\text { Value }\end{array}$ & $\begin{array}{c}\text { Overall } \\
\text { Diagnostic } \\
\text { Accuracy }\end{array}$ \\
\hline Mean & Decrease & $7 / 15$ & $6 / 13$ & .8 & $50 \%$ \\
\multirow{3}{*}{ 10th percentile } & Plateau/increase & $8 / 15$ & $7 / 13$ & & \\
& Decrease & $3 / 15$ & $12 / 13$ & $<.001$ & $86 \%$ \\
& Plateau/increase & $12 / 15$ & $1 / 13$ & & \\
\hline
\end{tabular}

\section{Quantitative Assessment}

The overall $\mathrm{ADC}_{\text {mean }}$ values were lower in patients who were ultimately shown to have tumor progression compared with patients with stable tumors (1.68 \pm 0.23 versus $2.04 \pm 0.78$ ), though the difference was not statistically significant $(P=.13)$. The average of $\mathrm{ADC}_{10}$ values
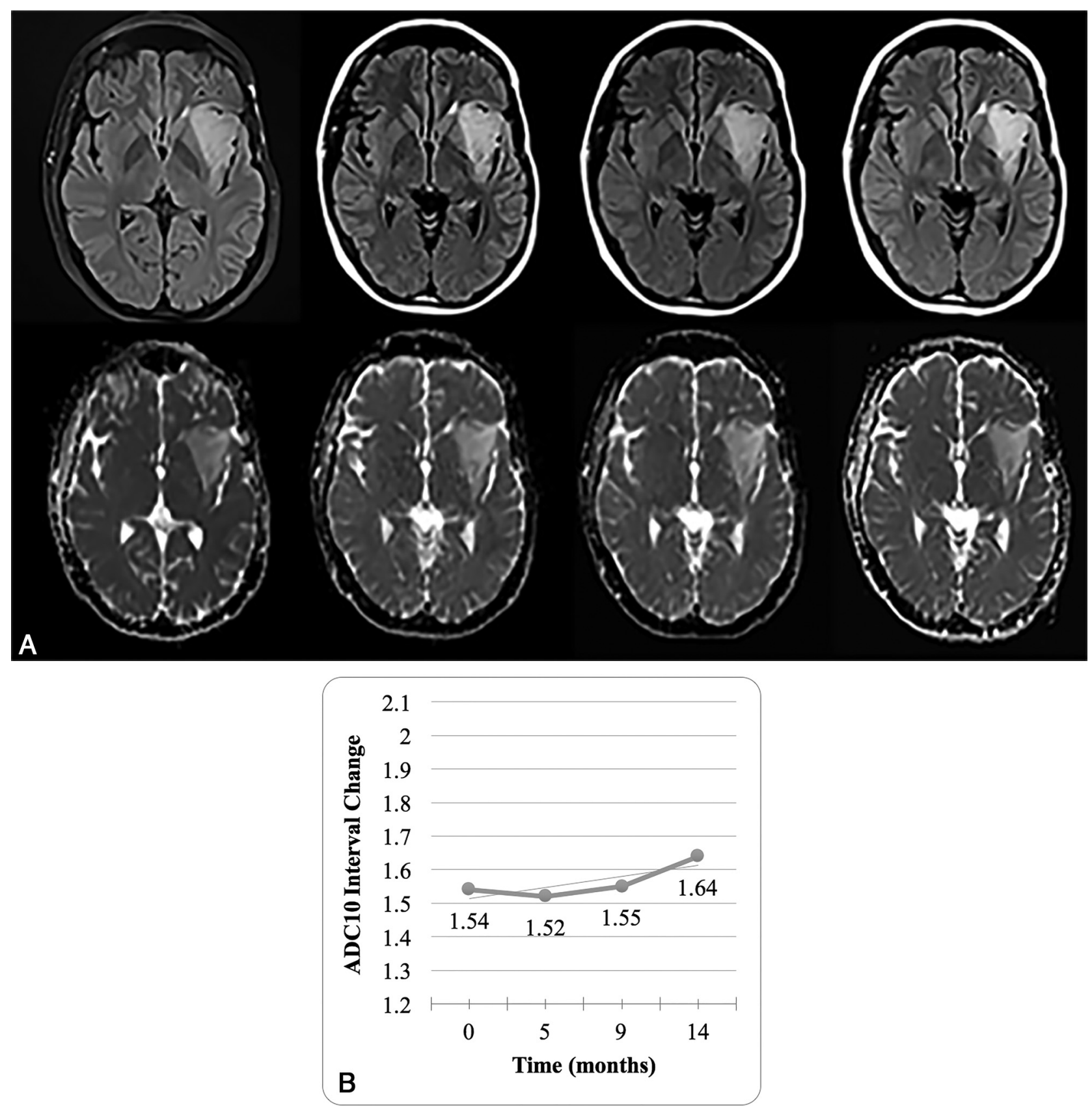

FIG 3. A 34-year-old woman diagnosed with grade II oligodendroglioma. A, Axial FLAIR/ADC images from 4 sequential MR images are shown (upper row: FLAIR; lower row: ADC; from left to right: first scan after initial diagnosis and at 5, 9, and 14 months). Review of FLAIR images shows stable extent of tumor during the entire follow-up. $B$, Plotting the normalized $A D C_{10}$ values demonstrates no significant decrease in $A D C_{10}$ values, concordant with stability of the tumor. 

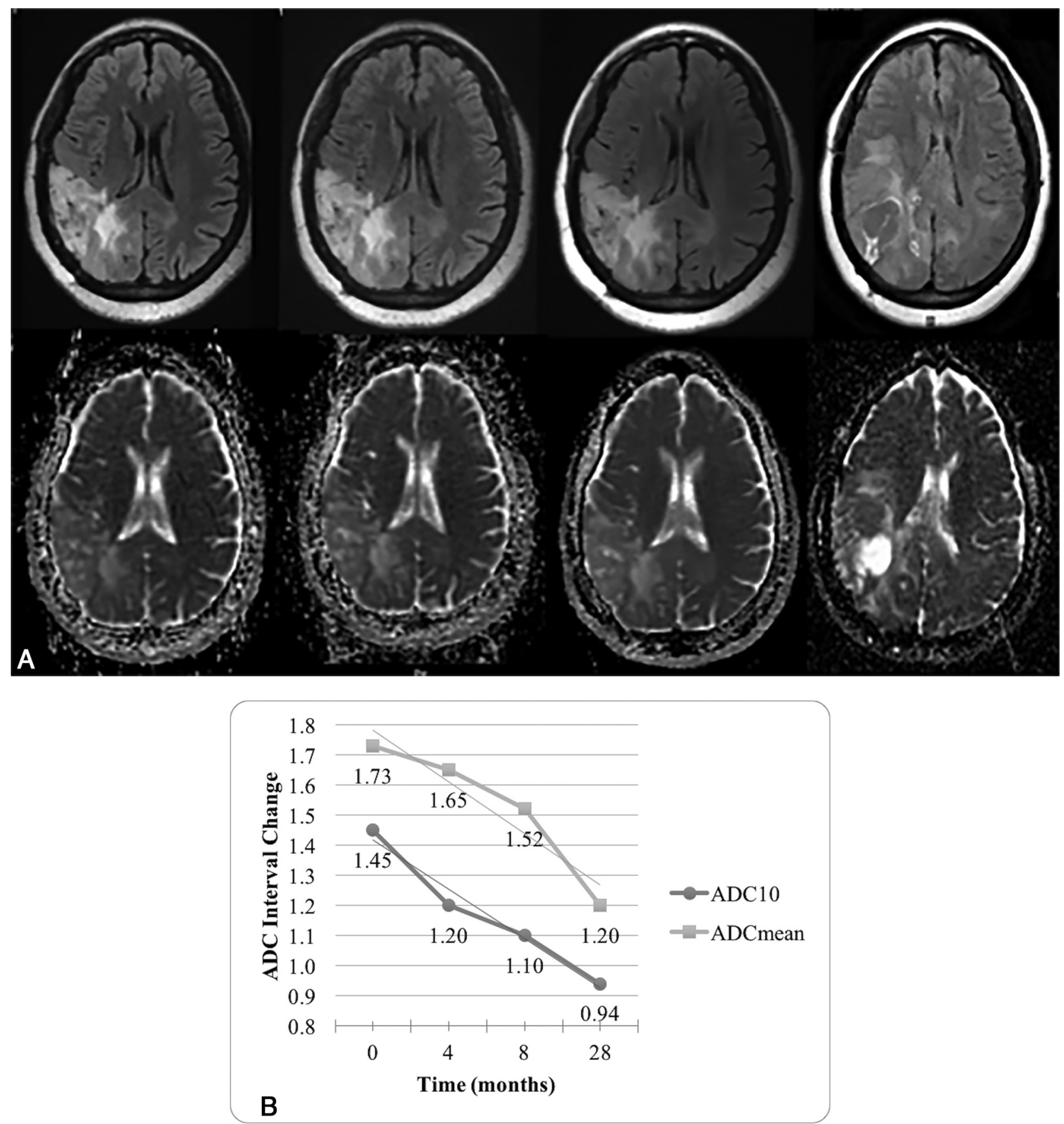

FIG 4. A 73-year-old woman diagnosed with a grade II oligodendroglioma. A, Axial FLAIR/ADC images from 4 sequential scans are shown (upper row: FLAIR; lower row: ADC; from left to right: first scan after initial diagnosis and at 4, 8, and 28 months). Disease progression was diagnosed at 28 months on conventional imaging and confirmed by surgical pathology. $B, B_{0}$ th normalized $A D C_{10}$ and $A D C_{\text {mean }}$ ratios are plotted, demonstrating an interval decrease of $A D C_{10}$ as early as 4 months following baseline examination, indicating eventual tumor progression. $C$, Histograms of quantitative normalized ratios of $A D C_{10}$ and $A D C_{\text {mean }}$ for each MR imaging examination.

was significantly $(P=.03)$ lower in patients with ultimate tumor progression compared with patients with stable tumors $(1.21 \pm$ 0.24 versus $1.49 \pm 0.37, P=.03)$. ROC analysis showed sensitivity/specificity and overall diagnostic accuracy of $84.6 \% / 40 \% /$ $62.3 \%$ (95\% CI, 0.42-0.80) for $\mathrm{ADC}_{\text {mean }}$ at a threshold of 1.8 and $46.2 \% / 93.3 \% / 69.7 \%$ (95\% CI, 0.55-0.89) for $\mathrm{ADC}_{10}$ at a threshold of 1.1 .

If one applied the Fisher exact test for analysis of disease progression using the sequential ADC interval change score, the in- terval change in $\mathrm{ADC}_{\text {mean }}$ matched the expected pattern in $6 / 13$ patients with tumor progression and $8 / 15$ patients with stable disease (sensitivity, 53.3\%; specificity, $46.2 \%$; overall diagnostic accuracy, $49.7 \%)$. The interval change in the $\mathrm{ADC}_{10}$ score matched the expected pattern in $12 / 13$ patients with tumor progression and $12 / 15$ patients with stable disease (sensitivity, $92.3 \%$; specificity, $80 \%$; overall diagnostic accuracy, 86.2\%). The ORs for identifying tumor progression were $1(P=.90)$ for $\mathrm{ADC}_{\text {mean }}$ and $48(P=.001)$ for $\mathrm{ADC}_{10}$, respectively. Table 2 summarizes the 


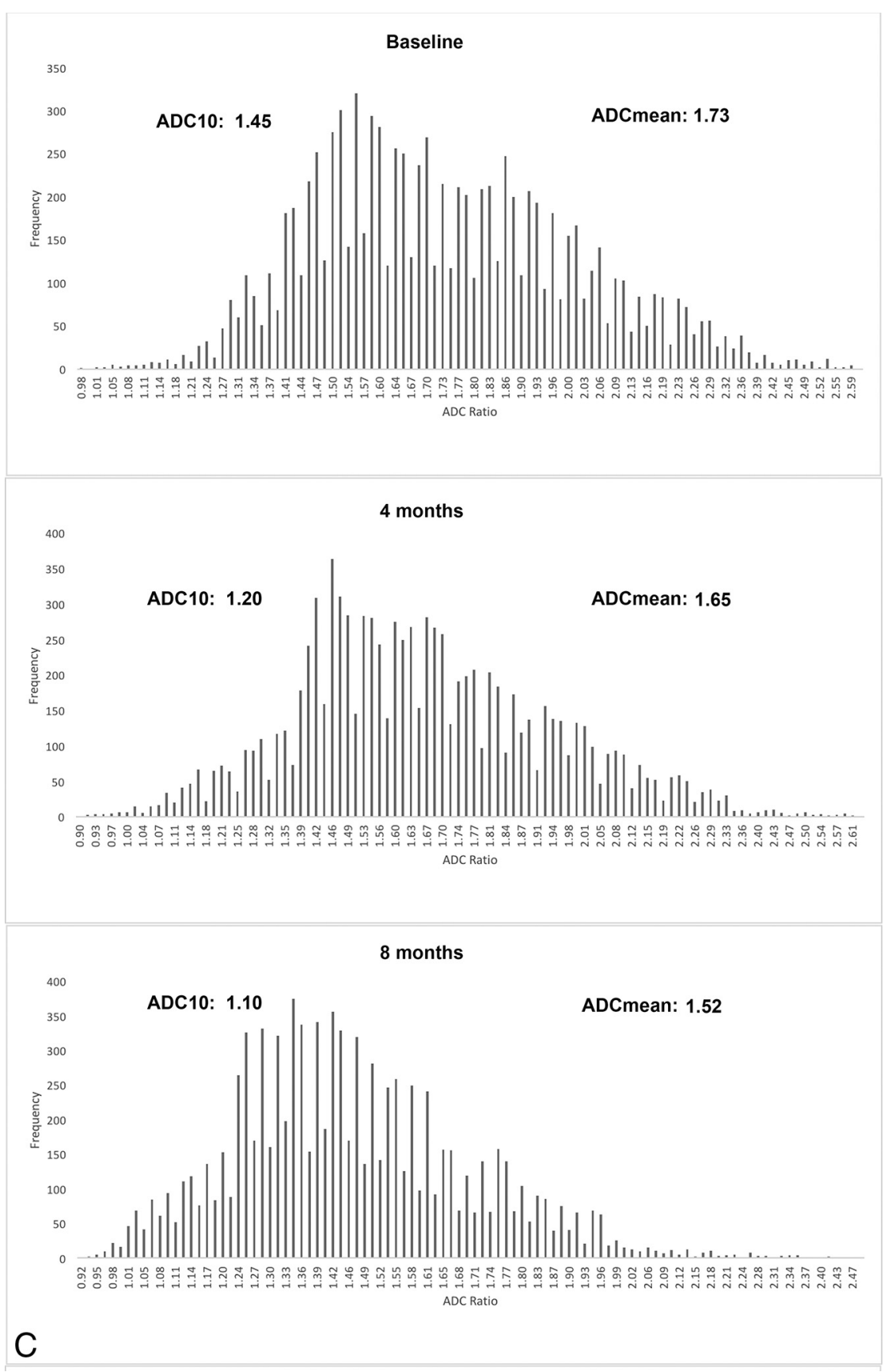

FIG 4. Continued.

results and diagnostic accuracy of the ADC interval change in identifying progression-versus-stable tumor.

Figure 3 shows an example of a stable pattern of $\mathrm{ADC}_{10}$ interval change in a patient who remained stable through the follow-up.

In $12 / 13$ patients with radiologic progression, the $\mathrm{ADC}_{10}$ interval change correctly predicted progression, on average, 8 months (range, 4.1-15.1 months) before the lesions progressed on conventional imaging on consensus interpretation. In 3 patients who were determined to have stable disease, an interval change in $\mathrm{ADC}_{10}$ incorrectly pointed to progression approximately 4.8 months (range, 3-6 months) earlier than conventional sequences. Figure 4 shows an example of how the interval decrease in $\mathrm{ADC}_{10}$ can identify tumor progression earlier than conventional imaging.

All our patients who demonstrated radiologic progression $(n=13)$ underwent a second surgical resection and/or biopsy. Higher grade transformation (grades III and IV) was found in 8 patients, while 5 patients maintained their initial grade despite a progressive increase in size and extent of the tumor.

The overall $\mathrm{ADC}_{\text {mean }}$ values were $1.70 \pm 0.22$ and $1.63 \pm 0.25$ in patients with stable grades compared with patients with higher grade transformation, respectively $(P=.64)$. ROC analysis was not significant $(P=.62)$ in differentiating stable-versus-higher grade transformation (area under the curve, 0.62; sensitivity, $50 \%$; specificity, $83.3 \%$; $95 \% \mathrm{CI}$, 0.33-0.86). The average of $\mathrm{ADC}_{10}$ values was $1.36 \pm 0.18$ and $1.16 \pm 0.22$ in patients with stable grades compared with patients with higher grade transformation, respectively $(P=.09)$. ROC analysis did not reach statistical significance $(P=.07)$ in differentiating stableversus-higher grade transformations (area under the curve, 0.75; sensitivity, $87.5 \%$; specificity, $66.7 \%$; $95 \%$ CI, $0.46-0.94)$.

\section{DISCUSSION}

ADC values of glioma tumors inversely correlate with tumor cellular density. ${ }^{10,11}$ This correlation has been studied as a possible means of identifying and grading LGGs, ${ }^{12-15}$ as well as monitoring the response to treatment. ${ }^{16,17}$ In this study, we demonstrated that a progressive downward trend of $\mathrm{ADC}_{10}$ interval change can predict tumor progression in patients with LGGs and before apparent radiologic progression on conventional MR imaging.

Our results show that the interval change of $\mathrm{ADC}_{10}$ is superior in the early detection of LGG progression in comparison with single-time-point quantitative ADC values. We demonstrated that the interval change in the $\mathrm{ADC}_{10}$ score matched the expected pattern in 12/13 patients with tumor progression and 12/15 patients with stable disease, yielding an overall diagnostic accuracy of $86 \%$. Using $\mathrm{ADC}_{10}$ interval change, we detected progression, on average, approximately 8 months before it was apparent on conventional imaging. Using single-time-point ADC values and traditional threshold methodology resulted in only a modest diagnostic performance, with an overall diagnostic accuracy of $62.3 \%$ at a threshold of 1.8 for $\mathrm{ADC}_{\text {mean }}$ and $69.7 \%$ at a threshold of 1.1 for $\mathrm{ADC}_{10}$.

The superiority of evaluating interval ADC changes instead of 
set threshold ADC values may be explained, in part, by the heterogeneous nature of LGG tumors. Specifically, tumor heterogeneity with existing variable and mixed tissue patterns due to the spatial variation in their cellular density, microenvironmental factors, and water content may result in varying quantitative ADC values across these tumors. By evaluating the interval change in $\mathrm{ADC}$ values in a particular lesion, the heterogeneity inherent in the individual tumor type can be internally controlled, resulting in an "individualized correction" that helps minimize confounding pathophysiologic factors.

Another reason for the modest performance of threshold methodology is the heterogeneity of scanners used in our study, which is an inherent limitation for any clinical research performed in a large institution using multiple MR imaging scanners. Despite the use of standardized imaging protocol and parameters, variability related to different MR imaging gradient systems and calibrations, software packages, and image scaling remains a major source of errors in quantitative image analysis such as ours. ${ }^{19}$

We also showed that $\mathrm{ADC}_{10}$ was predictive of LGG progression, whereas $\mathrm{ADC}_{\text {mean }}$ was not. In contrast to mean values that can be diluted by tissue and microenviromental heterogeneity, the lowest 10th percentile value was chosen to represent regions of tumor with the highest cellular density and proliferation. This is consistent with prior reports showing that the lowest representation of ADC using fifth percentile ${ }^{20}$ or 10th percentile ADC val$u^{2} s^{21,22}$ has a higher correlation with tumor grade and cellular density in comparison with $\mathrm{ADC}_{\text {mean }}$ values.

One limitation of this study was the small sample size, and a larger cohort would be needed to further validate our results. For example, in our patients with higher grade transformation, we found lower values of $\mathrm{ADC}_{10}$ in comparison with patients with stable tumor; however, this difference did not reach statistical significance $(P=.09)$, despite prior promising results. ${ }^{12,13}$ Failure to demonstrate a significant difference may be a consequence of our sample size. The retrospective nature of our study could also introduce unknown errors including, but not limited to, selection and recall bias. Like many other studies, for quantitative analysis, we relied on the segmentation of tumor, which can introduce errors due to varying expertise and subjectivity of the observer. ADC values derived from regional ROIs can underestimate the heterogeneity of tumoral density ${ }^{23}$ and are prone to operatordependent sampling bias. ${ }^{24}$ To minimize this variability, we used a semiautomated segmentation to create VOIs based on FLAIR hyperintense tumor. This VOI methodology has been shown to be more reproducible compared with other segmentation methods such as ROI-based analysis. ${ }^{25}$ Quantitative analysis of ADC values may vary among different institutions depending on the variety of imaging parameters, type of scanner hardware and software, and image postprocessing. Therefore, the results should be interpreted within this context. We tried to minimize these errors using a standardized imaging protocol and parameters and also normalizing the ADC values to the contralateral side for the MR imaging study. We also performed a weekly calibration of our scanners using a diffusion phantom. Nevertheless, these confounding factors are inevitable for longitudinal clinical studies such as ours in which several MR imaging scanners were used.

\section{CONCLUSIONS}

The interval change of $\mathrm{ADC}_{10}$ values can be used to identify progression of LGGs with a diagnostic accuracy of $86 \%$ and before apparent radiologic progression on conventional MR imaging. Further investigation with larger patient populations is required to confirm these findings. If its value is established in a larger prospective clinical study, $\mathrm{ADC}_{10}$ has the potential to be used for prediction of LGG progression earlier in the disease course when radiologic progression is not apparent on FLAIR or T1 postcontrast imaging.

Disclosures: Bradley N. Delman—UNRELATED: Consultancy: Bayer HealthCare Pharmaceuticals, Comments: money paid to the institution; Payment for Lectures Including Service on Speakers Bureaus: Bayer HealthCare Pharmaceuticals; Travel/ Accommodations/Meeting Expenses Unrelated to Activities Listed: Bayer HealthCare Pharmaceuticals. Kambiz Nael—UNRELATED: Board Membership: Olea Medical, Comments: Medical Advisory Board.

\section{REFERENCES}

1. Delgado-López PD, Corrales-García EM, Martino J, et al. Diffuse low-grade glioma: a review on the new molecular classification, natural history and current management strategies. Clin Transl Oncol 2017;19:931-44 CrossRef Medline

2. Larsen J, Wharton SB, McKevitt F, et al. 'Low grade glioma': an update for radiologists. Br J Radiol 2017;90:20160600 CrossRef Medline

3. Nahed BV, Redjal N, Brat DJ, et al. Management of patients with recurrence of diffuse low grade glioma: a systematic review and evidence-based clinical practice guideline. J Neurooncol 2015;125: 609-30 CrossRef Medline

4. van den Bent MJ, Wefel JS, Schiff D, et al. Response assessment in neuro-oncology (a report of the RANO group): assessment of outcome in trials of diffuse low-grade gliomas. Lancet Oncol 2011;12: 583-93 CrossRef Medline

5. Fouke SJ, Benzinger T, Gibson D, et al. The role of imaging in the management of adults with diffuse low grade glioma: a systematic review and evidence-based clinical practice guideline. J Neurooncol 2015;125:457-79 CrossRef Medline

6. Asari S, Makabe T, Katayama S, et al. Assessment of the pathological grade of astrocytic gliomas using an MRI score. Neuroradiology 1994;36:308-10 CrossRef Medline

7. Kim MM, Lawrence TS, Cao Y. Advances in magnetic resonance and positron emission tomography imaging: assessing response in the treatment of low-grade glioma. Semin Radiat Oncol 2015;25:172-80 CrossRef Medline

8. Pallud J, Capelle L, Taillandier L, et al. Prognostic significance of imaging contrast enhancement for WHO grade II gliomas. Neuro Oncol 2009;11:176-82 CrossRef Medline

9. Vos MJ, Uitdehaag BM, Barkhof F, et al. Interobserver variability in the radiological assessment of response to chemotherapy in glioma. Neurology 2003;60:826-30 CrossRef Medline

10. Hayashida Y, Hirai T, Morishita S, et al. Diffusion-weighted imaging of metastatic brain tumors: comparison with histologic type and tumor cellularity. AJNR Am J Neuroradiol 2006;27:1419-25 Medline

11. Chenevert TL, Stegman LD, Taylor JM, et al. Diffusion magnetic resonance imaging: an early surrogate marker of therapeutic efficacy in brain tumors. J Natl Cancer Inst 2000;92:2029-36 CrossRef Medline

12. Cui Y, Ma L, Chen X, et al. Lower apparent diffusion coefficients indicate distinct prognosis in low-grade and high-grade glioma. J Neurooncol 2014;119:377-85 CrossRef Medline

13. Hathout L, Ellingson BM, Cloughesy TF, et al. Patient-specific characterization of the invasiveness and proliferation of low-grade gliomas using serial MR imaging and a mathematical model of tumor growth. Oncol Rep 2015;33:2883-88 CrossRef Medline

14. Kitis $\mathrm{O}$, Altay H, Calli C, et al. Minimum apparent diffusion coeffi- 
cients in the evaluation of brain tumors. Eur J Radiol 2005;55:393400 CrossRef Medline

15. Murakami R, Hirai T, Sugahara T, et al. Grading astrocytic tumors by using apparent diffusion coefficient parameters: superiority of a one- versus two-parameter pilot method. Radiology 2009;251: 838-45 CrossRef Medline

16. Hein PA, Eskey CJ, Dunn JF, et al. Diffusion-weighted imaging in the follow-up of treated high-grade gliomas: tumor recurrence versus radiation injury. AJNR Am J Neuroradiol 2004;25:201-09 Medline

17. Higano S, Yun X, Kumabe T, et al. Malignant astrocytic tumors: clinical importance of apparent diffusion coefficient in prediction of grade and prognosis. Radiology 2006;241:839-46 CrossRef Medline

18. Brasil Caseiras G, Ciccarelli O, Altmann DR, et al. Low-grade gliomas: six-month tumor growth predicts patient outcome better than admission tumor volume, relative cerebral blood volume, and apparent diffusion coefficient. Radiology 2009;253:505-12 CrossRef Medline

19. Chenevert TL, Malyarenko DI, Newitt D, et al. Errors in quantitative image analysis due to platform-dependent image scaling. Transl Oncol 2014;7:65-71 CrossRef Medline
20. Kang Y, Choi SH, Kim YJ, et al. Gliomas: histogram analysis of apparent diffusion coefficient maps with standard- or high-b-value diffusion-weighted MR imaging-correlation with tumor grade. Radiology 2011;261:882-90 CrossRef Medline

21. Wen Q, Jalilian L, Lupo JM, et al. Comparison of ADC metrics and their association with outcome for patients with newly diagnosed glioblastoma being treated with radiation therapy, temozolomide, erlotinib and bevacizumab. J Neurooncol 2015;121:331-39 CrossRef Medline

22. McDonald CR, Delfanti RL, Krishnan AP, et al. Restriction spectrum imaging predicts response to bevacizumab in patients with highgrade glioma. Neuro Oncol 2016;18:1579-90 CrossRef Medline

23. Cha S. Update on brain tumor imaging: from anatomy to physiology. AJNR Am J Neuroradiol 2006;27:475-87 Medline

24. Tozer DJ, Jäger HR, Danchaivijitr N, et al. Apparent diffusion coefficient histograms may predict low-grade glioma subtype. NMR Biomed 2007;20:49-57 CrossRef Medline

25. Jung SC, Choi SH, Yeom JA, et al. Cerebral blood volume analysis in glioblastomas using dynamic susceptibility contrast-enhanced perfusion MRI: a comparison of manual and semiautomatic segmentation methods. PLoS One 2013;8:e69323 CrossRef Medline 